\title{
Bioabsorbable Bone Plates Enabled With Local, Sustained Delivery of Bmp2 for Bone Regeneration
}

\author{
Young Wook Jo*1,2 and Min Gee Chung ${ }^{3}$ \\ ${ }^{1}$ Department of Biotechnology, Yonsei University, Korea \\ ${ }^{2}$ Laboratory of Pharmaceuticals, Biostandard, Korea \\ ${ }^{3}$ Korea International School (KIS), Korea
}

Received: December 14, 2017; Published: February 13, 2018

*Corresponding author: Young Wook Jo, Department of Biotechnology, College of Engineering, Yonsei University, Seoul 03722, Republic of Korea, Email: carpediemwj@gmail.com

\begin{abstract}
We prepared a bone plate enabled with the local, sustained release of BMP2, which is a drug known to inhibit Osteoclastic-mediated bone resorption and also expedite the bone-remodeling activity of osteoblasts. For this, we coated a bone plate already in clinical use (PLT-1031, Inion, Finland) with a blend of BMP2 and a biocompatible polymer, 4-azidobenzoic acid-modified chitosan (i.e., Az-CH) photo-cross linked by UV irradiation. As we performed the in vitro drug release study, the drug was released from the coating at an average rate of $4.03 \mu \mathrm{g} / \mathrm{day}$ for 63 days in a sustained manner. To examine the effect on bone regeneration, the plate was fixed on an $8 \mathrm{~mm}$ cranial critical size defect in living rats and the newly formed bone volume was quantitatively evaluated by micro-computed tomography (micro-CT) at scheduled times over 8 weeks. At week 8, the group implanted with the plate enabled with sustained delivery of BMP2 showed a significantly higher volume of newly formed bone $(52.78 \pm 6.84 \%)$ than the groups implanted with the plates without drug $(23.6 \pm 3.81 \%)$ ( $\mathrm{p} \mathrm{b} 0.05)$. The plate enabled with BMP2 delivery also exhibited good biocompatibility on H\&E staining, which was comparable to the Inion plate already in clinical use. Therefore, we suggest that a bone plate enabled with local, sustained delivery of BMP2 can be a promising system with the combined functionality of bone fixation and its expedited repair.
\end{abstract}

Abbreviations: PLGA: Poly Lactic-Co-Glycolic Acid; PLA: Poly Lactic Acid; PGA: Poly Glycolic Acid; FDA: Food and Drug Administration; ACDF: Anterior Cervical Discectomy and Fusion; PEI: Poly Ethylenimine; PEG: Poly Ethylene Glycol; SEM: Scanning Electron Microscopy; UTS: Ultimate Tensile Strength; UTM: Universal Testing Machine; ELISA: Enzyme-Linked Immunosorbent Assay; DMEM: Dulbecco's Modified Eagle Medium

\section{Introduction}

Bone fixation systems made of biodegradable polymers, such as poly (lactic-co-glycolic acid) (PLGA), poly (lactic acid) (PLA) or poly (glycolic acid) (PGA), have attracted a great deal of interest as they would not need a secondary removal surgery due to biodegradability $[1,2]$. The major compartments of the bone fixation systems are plates and screws, where the plate is positioned and fixed on a fractured bone by screws. In this way, the undesired motion of the fractured bone can be prevented until complete healing. However, the bone fixation systems currently in clinical use do not have the functionality to treat patients with bone loss or diseases, such as osteoporosis. It was reported that approximately $11.4 \%$ of comminuted fractures induce bone loss [3], which would impair the stability of the bone fixation systems, thereby often needing a secondary surgery [3,4]. Such complications would also include infection, which was reported to occur in approximately $13.9 \%$ patients with fractured bone [5]. The osteoporotic patients often show low bone density, which delays bone healing [6] and causes severe complications, such as microfracture, malunion or a loosening of the fixation system even with adequate fixation of fractured bone $[7,8]$. It has been reported that the failure of bone fixation systems occurs in 2 to $10 \%$ of fractures related to osteoporosis $[8,9]$.

For these reasons, a strategy to facilitate bone healing is needed to properly treat fractured bone without a failure of the fixation system [10]. BMP-2, a member of TGF-b super family has potential to differentiate pluripotent cell to osteoblasts and is a key molecule for bone homeostasis. After rhBMP-2 was approved by Food and Drug Administration (FDA) as an osteoinductive bone graft substitutes (In FUSE, Medtronic Sofamor Danek), BMP-2 has been widely used in the lumbar inter body fusion, open tibial fracture and oral- maxillofacial surgery[11,12]. Currently, rhBMP-2 soaked in absorbable collagen sponge scaffold loaded in LT Cage taperd fusion device is the only instruction for bone graft use. With enhanced retention of rhBMP-2 and achievement of bone regeneration however, off-label use of rhBMP-2 in improper location and dosage has also emerged $[13,14]$. Because of these off-label uses of rhBMP- 
2, many and various complications have reported including lifethreatening swelling after anterior cervical discectomy and fusion (ACDF), as well as seroma, vertebral osteolysis and ectopic bone formation $[15,16]$. These adverse effects are thought that because of short half-life of BMP-2 in serum condition and limitation of proper delivery system.

RhBMP-2 has been used in high dosage to achieve satisfactory bone regeneration [17]. To solve these unwanted problems, various kinds of carriers have been developed to fulfill controlled and more effective delivery of rhBMP-2. Chemical polymers such as Polyethylenimine (PEI), polyethylene glycol (PEG) [18], poly (lactic-co-glycolic acid) (PLGA) [19] and natural polymers such as collagen [20], albumin, chitosan [21] and silk fibroin [22] have been widely used with different formats, not only for rhBMP-2 delivery but also other bioactive molecules. However, futher studies are still nessasary for application of these systems in clinical use [23]. In this work, therefore, we prepared a bone fixation plate with the added functionality of the local, sustained delivery of BMP2. For this, we employed a bone plate already in clinical use (PLT-1031, Inion, Finland) and coated it with 4-azidobenzoic acid-modified chitosan (i.e., Az-CH) loaded with BMP2. Az-CH was cross linked via UV irradiation to serve as a drug diffusion barrier for the sustained delivery of BMP2 [24,25]. In addition, cross linked Az-CH can form covalent bonds with poly(lactic acid), one of the major constituents of the Inion plate [26,27], and thus, the Az-CH based coating could be stably attached on the surface of the Inion plate.

$\mathrm{Az}-\mathrm{CH}$ is also proven to be biocompatible to a large extent $[27,28]$. We characterized the morphology and coating by scanning electron microscopy (SEM). We also performed the in vitro drug release study in phosphate buffered saline (PBS; pH 7.4) at $37{ }^{\circ} \mathrm{C}$ with the plate coated with both Az-CH and BMP2. For the in vivo evaluation, the plates were fixed on a craniotomy defect, $8 \mathrm{~mm}$ in diameter, created on the skull of living rats [29]. The degree of reconstructed bone volume was quantitatively measured using micro-computed tomography (micro-CT) at a predetermined schedule for 8 weeks following implantation. The histopathologic analyses were also carried out with the tissue including the implanted plate 8 weeks after implantation with hematoxylin and eosin (H\&E) staining.

\section{Materials and Methods}

\section{Materials}

4-azidobenzoic acid was obtained from Tokyo Chemical Industry (Tokyo, Japan). Chitosan (Mw; b $200 \mathrm{kDa}$, degree of deacetylation; $\quad 75-85 \%), \quad \mathrm{N}, \mathrm{N}, \mathrm{N}^{\prime}, \mathrm{N}^{\prime}$-tetramethylethylenediamine (TEMED), 1-ethyl- 3-(3-dimethylaminopropyl)-carbodiimide (EDC), o-phthaldialdehyde (OPA) and 2- mercaptoethanol (2 ME) were all purchased from Sigma-Aldrich (MO, USA). Phosphate buffered saline (PBS, pH 7.4) was obtained from the Seoul National University Hospital Biomedical Re- search Institute. Bioabsorbable bone fixation plates (PLT-1031), composed of poly (trimethylene carbonate), polylactide and polyglycolide [30], and were purchased from Inion (Finland). Zolazepam and tiletamine $(0.3 \mathrm{ml} / \mathrm{kg}$; Zoletil-->) were supplied from Virbac (France). Xylazine $(0.1 \mathrm{ml} /$ kg; Rompun-->) was obtained from Bayer (Germany).

\section{Synthesis of an 4-Azidobenzoic Acid-Modified Chitosan (Az-CH) and Loading BMP2 Protein}

Az-CH was synthesized as described in a previous study. In brief, a chitosan solution was first prepared with $200 \mathrm{mg}$ chitosan dissolved in $15 \mathrm{ml}$ distilled water adjusted to $\mathrm{pH} 4.75$ using acetic acid solution. TEMED (116.2mg) was dissolved in $1 \mathrm{ml}$ distilled water, which was then added to the chitosan solution. To this resulting solution, a mixed solution of EDC in $1 \mathrm{ml}$ distilled water and $40 \mathrm{mg} 4$-azidobenzoic acid in $1 \mathrm{ml}$ dimethyl sulfoxide was then added. After adjusting the $\mathrm{pH}$ to 5 with $1 \mathrm{M} \mathrm{HCl}$, the reaction was carried out at room temperature over-night. The solution was filtered via a $0.22 \mu \mathrm{m}$-pore filter (GSWP04700, Millipore, Bedford, $\mathrm{MA}$ ), which was then freeze-dried for 3 days to give a dry powder of Az-CH.

For loading the BMP2 protein, we first cut a whole piece of an Inion bone plate (PLT-1031, mesh type: $14 \times 14$ holes) into squareshaped pieces $(6 \times 6 \mathrm{~mm})$, each with a screw hole at the center. These pieces were each used as the UP samples without further treatment. Recombinant human BMP-2 was kindly provided from Deawoong Phamaceutical Co., Ltd., Seoul, Korea. RhBMP-2 was added to the mixture after vortexed briefly. To prepare the coated samples, i.e., Az-CH_P or BMP-Az-CH_P, the coating solution was first prepared: $20 \mathrm{mg} \mathrm{Az}-\mathrm{CH}$ or a blend of $20 \mathrm{mg} \mathrm{Az}-\mathrm{CH}$ and $2 \mathrm{mg}$ BMP2 was dissolved in $1 \mathrm{ml}$ of albumin buffered saline. Then, four drops of the coating solution ( $3 \mu \mathrm{l}$ per drop) were added on top of the unmodified plate around the screw hole at the center. We prepared the three different types of bone plate samples in this work:

a) UP: unmodified bone plates with no treatment

b) Az-CH_P: bone plates coated with Az-CH only

c) BMP-Az-CH_P: bone plates coated with Az-CH and BMP2

The coated samples were each placed under UV irradiation (100 W; 365nm, Blak-Ray, UVP, USA) for $5 \mathrm{~min}$ to crosslink Az-CH, which were then dried at room temperature for $24 \mathrm{~h}$ in a dark room (Figure 1B). In this work, we coated only one side of the plate that should be faced toward the fractured bone after fixation. We also avoided the screw holes from coating, which would be under severe frictional stress during fixation. For the in vivo experiments and the mechanical property evaluation, we cut the Inion plate into a piece containing three screw holes. The outer two holes were used to suture and fix the plate on a bone for the in vivo experiments or employed as sites for clamping for the mechanical property evaluation. The coatings were made in the same way as described above, only around the screw hole in the middle.

\section{Plate Characterizations}

The surface of the coating on the plate samples (i.e., BMP-Az$\left.\mathrm{CH}_{-} \mathrm{P}\right)$ was examined and compared with that of the non-coated, intact surface, using a scanning electron microscope (SEM; 7501F, 
Jeol, Japan). Prior to imaging, the sample was placed on the SEM specimen mount and sputter coated with platinum for $5 \mathrm{~min}$ (208HR, Cressington Scientific, England).

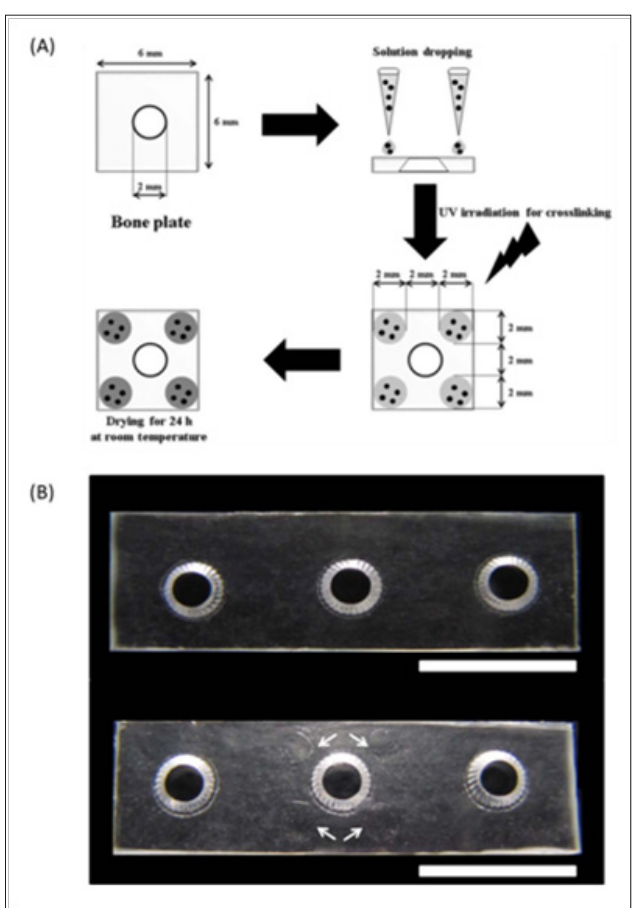

Figure 1: The Austenitic phase shape of the XP file $\left(35^{\circ} \mathrm{C}\right)$ (FKG Dentaire, La Chaux-de-Fonds, Switzerland).

\section{Mechanical Property Evaluation}

To examine the mechanical properties of the plate after coating, we measured the ultimate tensile strength (UTS) of the plate samples, i.e., UP, Az-CH_P and BMP-Az-CH_P. The plate samples were each loaded in a universal testing machine (UTM; Instron-5543, MA, USA) equipped with a load cell of $71 \mathrm{kN}$, where both ends of the sample were clamped and pulled at a rate of $3 \mathrm{~mm} / \mathrm{min}$ until they broke [31].

\section{Measurement of Drug-Loading Amount}

The amount of BMP2 in the coating was measured, following the method as previously employed [32]. The remaining rhBMP-2 in supernatant was measured for calculation of loading efficiency. RhBMP-2 was quantified by enzyme-linked immunosorbent assay (ELISA) kit (Antigenix America, Huntington Station, NY) according to the manufacturer's instructions. RhBMP-2 loading efficiency $=$ (actual loaded amount of rhBMP-2)-(remaining rhBMP-2 in supernatant) / (actual loaded amount

of rhBMP-2) x 100 (\%).

\section{In Vitro Drug Release Study}

The BMP-Az-CH_P was immersed in $2 \mathrm{ml}$ of phosphate buffered saline (PBS, pH 7.4) [33] at $37^{\circ} \mathrm{C}$, which was continuously agitated at $125 \mathrm{rpm}$ in a shaking incubator (SI-300, JEO TECH, Korea). For in vitro release study, BMP-Az-CH_P (total 5ug of rhBMP-2 included) was re-suspended in $1 \mathrm{ml}$ of Dulbecco's modified eagle medium
(DMEM) (HyClone, Logan, Utah) containing $10 \%$ FBS (HyClone, Logan, Utah), then placed in a rotator maintained at $37^{\circ} \mathrm{C}$. At given time points, samples were centrifuged $\left(10,000 \mathrm{x} \mathrm{g}, 10 \mathrm{~min}, 4{ }^{\circ} \mathrm{C}\right)$ and supernatant (released medium) was stored at $-80^{\circ} \mathrm{C}(\mathrm{n}=3)$ for further analysis. $1 \mathrm{ml}$ of fresh medium was replaced to the BMP-AzCH_P. Amount of released rhBMP-2 was determined by ELISA.

\section{In Vitro Cell Cytotoxicity Evaluation}

We used two distinct cell types (Korean Cell Line Bank, Korea), i.e., the NIH3T3 mouse fibroblasts cells, to assess the cytotoxicity of the plate samples prepared in this work. The NIH3T3 fibroblasts were grown in the media (WelGENE, Korea) of DMEM, respectively, both of which were supplemented with $10 \%$ fetal bovine serum (Gibco, Life Technologies, UK) and 1\% antibiotic (penicillin, 10,000 U ml-1; Gibco, Life Technologies, UK). The cells were incubated in an atmosphere of 5\% CO2 in air and 100\% relative humidity at 37 ${ }^{\circ} \mathrm{C}$. All samples were sterilized prior to the test using ethylene oxide gas. Three batches were tested for each of the sample types. For evaluation with NIH3T3 cells, the samples were each placed in a 24well cell culture plate where the NIH3T3 fibroblasts were prepared at a density of $2.0 \times 104$ cells/well. After incubation in a humidified atmosphere with $5 \% \mathrm{CO} 2$ at $37^{\circ} \mathrm{C}$ for $24 \mathrm{~h}$ (HERAcell $150 \mathrm{i}$, Thermo Scientific, USA), the samples were each washed with distilled water to fully remove the non-adhered cells. Afterward, the adhered cells on the coating were quantitatively investigated. For quantitative evaluation of the cells, the EZ-Cytox Cell viability assay kit was used, following the manufacturer's instruction (No. EZ- 1000, DAEILAB SERVICE, Korea) [34]. The absorbance was measured at 450nm using a micro plate reader (VERSA max, Molecular Devices, USA).

\section{In Vivo Animal Study}

For the in vivo evaluation of the plate samples, we used 8-week-old male SD rats weighing 250-300g (Koatech, Keung-Ki, Pyong-Taek, Korea). The rats were cared and housed, following the protocol approved by the Institutional Animal Care and Use Committee. The rats were provided with food and water ad libitum. All samples were sterilized prior to implantation using ethylene oxide gas. Three batches were tested for each of the sample types. To examine the bone regeneration effect, we first created the calvarial bone defect in rats as previously reported [35]. Anesthesia was induced by intramuscular injection of a cocktail of Zolazepam and tiletamine $(0.3 \mathrm{ml} / \mathrm{kg}$; Zoletil-->, Virbac, France), and xylazine $(0.1 \mathrm{ml} / \mathrm{kg}$; Rompun-->, Bayer, Germany). Then, the hair on the head was clearly shaved, followed by cleaning with Betadine--> (Hyundai Pharm, Korea) and the calvarial bone was exposed through a skin incision, $5 \mathrm{~cm}$ in length. A calvarial bone defect, $8 \mathrm{~mm}$ in diameter, was created with a trephine (TPHB-B8, Osung, Korea). To fix the plate in place, two holes for suturing, $10 \mathrm{~mm}$ apart, were also made by drilling, each of which was located $2 \mathrm{~mm}$ away from the boundary of a defect.

The surgical area was washed with saline to fully remove bone debris. The plate sample was placed on the calvarial bone defect with the coat- ed side facing toward the defect. Then, the plate was fixed with bio-absorbable suture (Vicryl 3-0, Ethicon, NJ, USA) 
through the outer two holes made for suturing. Then, the incised skin was closed with a bio-absorbable suture and a post-operative dressing was applied with Betadine-->. According to the types of the implanted samples, the animals were divided into four groups.

a) The no treatment group: the animals without the plate

b) The UP group: the animals implanted with the UP

c) The Az-CH_P group: the animals implanted with the AzCH_P

d) The BMP-Az-CH_P group: the animals implanted with the BMP-Az- CH_P Six animals were employed for each of the implant groups.

To evaluate new bone formation, images from the bone defect were obtained via micro-computed tomography (micro-CT; NFR Polaris-G90, In-vivo Micro-CT, NanoFocusRay, Korea) at 1, 4 and 8 weeks after plate implantation. Imaging was performed on the scanner at an isotropic voxel size of $9 \mu \mathrm{m}$ with an X-ray tube current of $180 \mu \mathrm{A}$ and a voltage at $55 \mathrm{kV}$. At each time for imaging, the animal was sedated with an intramuscular injection of Zoletil--> $0.3 \mathrm{mg} /$ $\mathrm{ml})$. From each of the micro- CT images, the regions of interest were defined, based on a cylindrical shape of the cranial defect made herein, where the new bone formation was determined by setting the gray threshold level at 200. The new bone volume percent was calculated by dividing the volume of newly formed bone by that of the initially created defect, using the AMIRA software (Version 5.4, ZIB \& Visage Imaging, Germany).

\section{Histologic and Histomorphometric Evaluation}

Eight weeks after plate implantation, three animals per group were sacrificed with carbon dioxide and the specimens including the bone defect and plate sample were biopsied. The specimen was fixed in $10 \%(\mathrm{v} / \mathrm{v})$ buffered neutral formalin for 3 days, which was then embedded with resin, sectioned into $30-40 \mu \mathrm{m}$ thick Slides (BS-3000 N, EXAKT, Germany) and stained with hematoxylin and eosin (H\&E). The slides were examined under an optical microscope (BX53F, OLYMPUS, Japan) with $\times 40$ and $\times 100$ magnifications for histologic evaluation by a professional pathologist. The images of the slides were also assessed for histomorphometric evaluation using Image J software (National Institute of Health, Bethesda, USA). The new bone area percent was obtained by dividing the area of newly formed bone by that of initially created defect.

\section{Statistics}

Mean percentages of new bone volumes and areas among the four different animal groups were statistically analyzed with oneway ANOVA with $\alpha=0.05$ followed by pair wise comparisons using a Tukey's post hoc test (Graph Pad Prism, version 5.01, USA). p < 0.05 was considered statistically significantly.

\section{Results}

As shown in (Figure 1), we briefly described the process of coating and loading the BMP2 protein in the plate. To confirm the formation of Az-CH, we assessed the UV spectra of $\mathrm{Az}-\mathrm{CH}$, chitosan and 4- azidobenzoic acid. For 4-azidobenzoic acid, an apparent peak at $266 \mathrm{~nm}$ was ascribed to the N3 group. This peak was also observed for Az-CH with a shift to $272 \mathrm{~nm}$, although the chitosan did not show any characteristic peaks in the spectrum [26]. This indicated that the reaction between a free amino group of the chitosan and a carboxyl group of 4-azidobenzoic acid indeed occurred to introduce the N3 group to the chitosan [27]. To further confirm this, we also assessed the FTIR spectra (Data not shown). (Figure 2) showed the scanning electron micrographs of the noncoated and coated surfaces of the BMP-Az-CH_P. The non-coated, unmodified surface was observed to be smooth with almost no micro-cracks or -defects (Figure 2A). On the other hand, a rough, wrinkled surface was observed on the coated surface (Figure 2B \& 2C), which appeared to be created while the coating solution was dried to leave the dry Az-CH. The surface morphology was not very different regardless of the presence of BMP2.
(A)

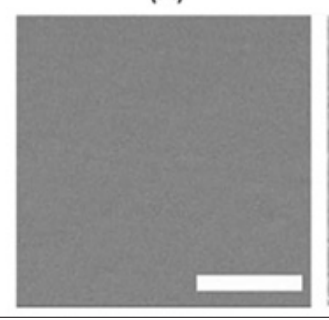

(B)

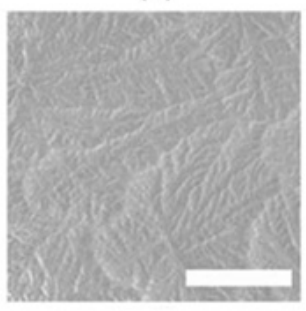

(C)

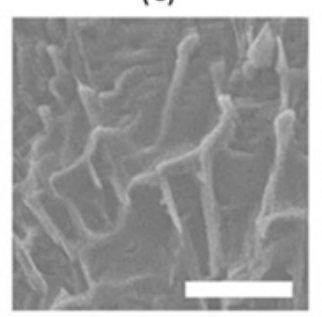

(D)

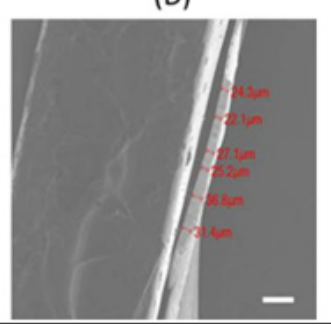

Figure 2: Scanning electron micrographs of the surfaces $(A)$ without and $(B, C)$ with the coating on the BMP-Az-CH_P. The scale bars represent (A, B) $100 \mu \mathrm{m}$ and (C) $10 \mu \mathrm{m}$. (D) Scanning electron micrograph of the side view of the coating on the BMP-Az-CH_P. The scale bar represents $100 \mu \mathrm{m}$.

The amount of chitosan in each coated plate was calculated to be about $0.18 \mathrm{mg}$ and the coating thickness in dry condition was measured to be 20-40 $\mu \mathrm{m}$ (Figure 2D). The coating appeared to be stable in PBS, showing almost no apparent sign of degradation until 120 days when visually observed. In contrary, with the presence of the serum environment [36-38], the coating was observed to gradually disappear, implying that the coating should degrade when implanted in vivo. Meanwhile, the Inion plate employed in this work is known to fully degrade in two years [39]. The bone plate needs to be strong enough to fix a fractured bone until healing [40-43]. This inherent mechanical property of the bone plate herein did not vary even after coating with the method employed in this work (Table 1). 
For the BMP-Az-CH_P, the loading amount of BMP2 was measured to be $293.84 \pm 8.04 \mu \mathrm{g}$ per plate (i.e., $73.46 \pm 2.01 \mu \mathrm{g}$ per coating). Considering a theoretical drug-loading amount of $300 \mu \mathrm{g}$, the BMPAz- $\mathrm{CH}_{-} \mathrm{P}$ exhibited a high drug loading efficiency of more than $97 \%$. This was somewhat expected as the coating herein was prepared simply by drying the drops of the drug and chitosan solution on a plate without any other treatment.

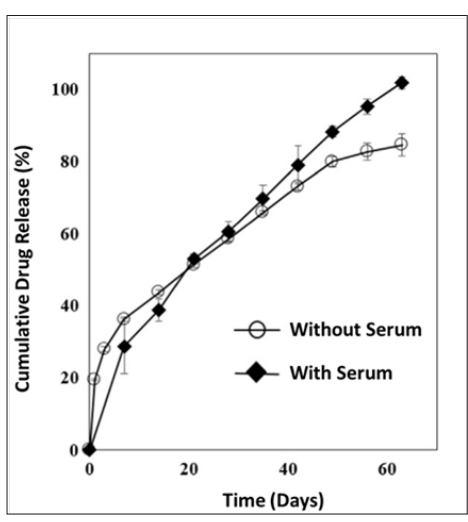

Figure 3: In vitro drug release profiles of the BMP-AzCH_P with and without serum.

Because of that, the drug was evenly distributed in the coating. According to the in vitro drug release study, the drug was released in a sustained manner for 63 days for both media with and without serum (Figure 3). Without serum, drug release was relatively fast during the first seven days with an approximate rate of 5.3\% per day (i.e., $15.56 \mu$ g per day), which slowed down to the rate of $0.9 \%$ per day (i.e., $2.6 \mu$ g per day) for the rest 56 days. With serum, drug release became faster than the one without serum after 28 days and almost $100 \%$ drug was released in 63 days. This long-term release could be ascribed to the electrostatic interactions between chitosan and BMP2 $[22,44]$. To evaluate the cytotoxicity of the coatings, we first quantitatively analyzed the number of NIH3T3 fibroblasts cells adhering to the surface of the plate samples, as shown in (Figure 4). The amount of adhered cells was not significantly different among the plate samples of the UP, Az- CH_P and BMP-Az-CH_P. Considering that the UP, an Inion bone plate in clinical use, is already proven to be safe to a large extent, those results suggested that the coatings prepared in this work are also not cytotoxic.

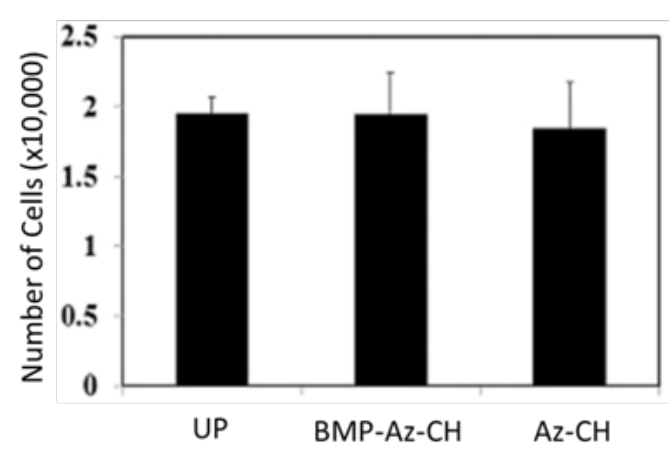

Figure 4: Cytotoxicity evaluation of the plate samples of the UP, Az-CH_P and BMP-Az-CH_P, using NIH3T3 fibroblasts.
To examine the efficacy of the BMP2-release coating, we investigated the degree of in vivo new bone formation within the calvarial bone defect, using micro-CT (Figure 5). For all animal groups, the average percent of new bone volume increased as the time elapsed. However, the degree of new bone formation was slow in the animal groups with-out BMP2 (i.e., the no treatment, UP, AzCH_P groups), where the new bone volume percent increased to at most $14-24 \%$ at 8 weeks and at all scheduled times of imaging, their values were not statistically significantly different. In contrast, the increase in new bone was evident with the BMP-Az-CH_P group. At 4 weeks, the new bone volume percent was $36.39 \pm 5.58 \%$, which increased further to $52.78 \pm 6.84 \%$ at 8 weeks. Notably, the average percent of new bone volume was statistically significantly higher with the BMP-Az-CH_P group from 4 weeks than those with the other different animal groups $(\mathrm{p}<0.05)$.

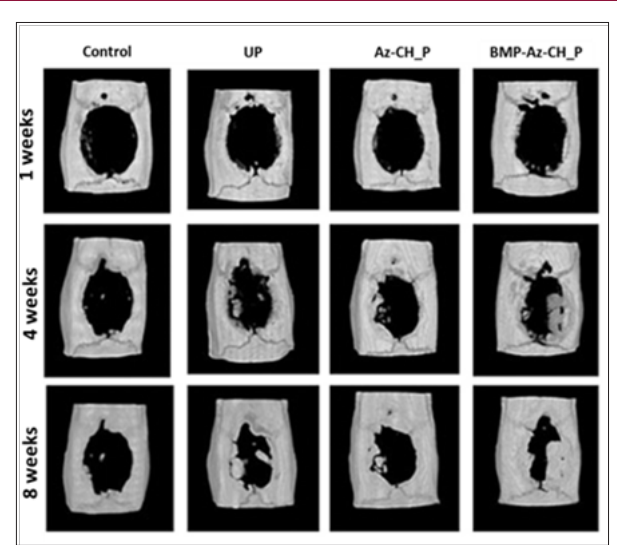

Figure 5: Micro-CT image analysis on new bone formation in cranial defects of the animal groups with no treatment and the ones fixed with the UP, Az-CH_P and BMP-AzCH_P at 1, 4 and 8 weeks after implantation.

The tissue of the calvarial defect was microscopically evaluated on H\&E staining 8 weeks after implantation, as shown in (Figure $6)$. In the no treatment group, only a few bone fragments were observed, mainly at the edge of the calvarial bone defect, which seemed to be newly formed after surgery. In the UP and Az-CH_P groups, fibrous tissue surrounded the plate sample, whereas newly formed bone tissue was scarce around the plate. In contrast, in the BMP-Az-CH_P group, the newly formed bone appeared to cover most of the surface of the plate and extended to the edges of the calvarial bone defect. At 8 weeks after surgery, for all groups, neutrophils, lymphocytes and other active inflammatory components were not observed around the plate and within the bone defect, where some amount of fibrous tissue was seen instead. Notably, the amount of fibrous tissue formation was grossly similar with all groups, i.e., the UP, Az-CH_P, and BMP-Az- CH_P groups, which were approximately $10-50 \mu \mathrm{m}$ in thickness from the plate.

These findings suggested that the extent of inflammatory reaction of the Az-CH_P and BMP-Az-CH_P was not significantly different from that of the UP, i.e., the Inion plate already in clinical use. To modulate such inflammation, a combined use of the formulation releasing antioxidants or anti-inflammatory drugs could be beneficial [45-47]. For example, the formulation 
in forms of microparticles could be easily embedded upon the coating during cross linking in this study. We also performed the histomorphometric analysis to quantitatively assess new bone formation. As shown in (Figure 7), the new bone area percent increased significantly with the BMP-Az-CH_P, compared with the other different groups. This result was consistent with the one from the micro-CT image analysis.

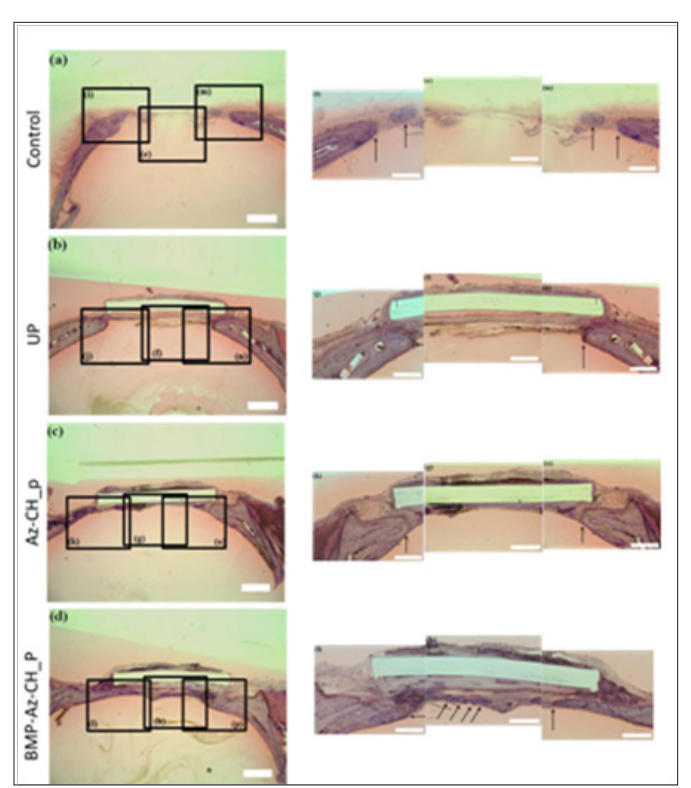

Figure 6: Histological images from the tissue around the cranial defect obtained at 8 weeks after implantation of the plate samples. The arrows indicate the specific locations of new bone formation. The scale bars represent $100 \mu \mathrm{m}$.

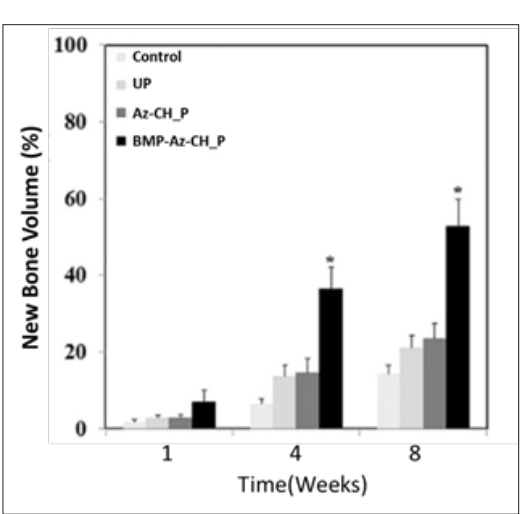

Figure 7: Histomorphometric analysis on new bone formation in cranial defects of the animal groups with no treatment and the ones fixed with the UP, Az-CH_P and BMP-Az-CH_P at 8weeks after implantation. *The ALAz-CH_P group was statistically significantly different from the other different groups.

\section{Discussion}

In this work, we suggested a bone plate enabled with a therapeutic ability is potentially advantageous for the treatment of bone loss associated with comminuted fractures as well as osteoporosis. For this, we prepared a proof-of-principle device, i.e., a bone plate coated with $\mathrm{Az}-\mathrm{CH}$ loaded with a osteogenic cytokine, BMP2. BMP2 is known to stimulate the recruitment and differentiation of osteoblasts, thereby facilitating new bone formation $[11,16]$. For this reason, the local, sustained delivery of BMP2 could be a promising way to expedite bone reconstruction. In this work, the BMP-Az-CH_P showed sustained drug release from the coating for 63 days at an average rate of approximately $4.0 \mu \mathrm{g}$ per day (Figure 5), which should be therapeutically effective in bone regeneration and also safe to a large extent, as many previous studies reported [48-50]. This long-term release of BMP2 appeared to allow suppression of the Osteoclastic activity, significantly mainly at the late stage of bone healing [29,51]. As a result, for the bone defect animal models herein, the BMP-Az-CH_P showed the enhanced in vivo new bone formation from 4 weeks, compared with the plate samples with-out BMP2 (Figures 5 \& 6). However, further study is needed to fully support the final clinical advantages of the BMP-Az-CH_P for treatment of fractures or bone loss with osteoporotic patients.

In this work, we employed a modified form of chitosan, Az$\mathrm{CH}$, as coating material. For the chitosan- based materials, to properly serve as drug diffusion barrier, chemical cross linking is often incorporated, using an agent such as glutaraldehyde, epichlorohydrin, glyoxal, etc. [52-54] However, this process requires a comparably long reaction time in an aqueous environment, which may degenerate the mechanical properties of the bone plate, originally needed for bone fixation. Un- like this, the Az-CH could form a cross linked network via UV irradiation in a short period of time ( $5 \mathrm{~min}$ ) to achieve the prolonged delivery of BMP2 $[25,52,55]$. Thus, the plate did not need to be exposed to an aqueous environment for a long time. Because of this, the plates herein appeared to retain their mechanical properties even after coating (Table 1). Most importantly, the Az-CH has been generally considered to be not cytotoxic [26,28], which could bealso supported by the results in this work (Figure 3). It has been reported that a high-concentrated use of BMP2 could induce adverse side effects and thus, there are recommended dose limitations [57]. Previously, the proposed systems for the local delivery of BMP2 were in form of scaffolds [22], grafts [58] and particles [23,59] and were evaluated to be effective to a large extent. However, for the treatment of bone fracture, those systems may require an additional procedure to apply them to the defected bone before or after implanting the bone fixation system during surgery. In this sense, a combined entity of the bone plate and drug delivery coating suggested in this work could be considered practically advantageous.

\section{Conclusion}

In this work, we suggest a bone plate enabled with therapeutic functionality of expedited bone regeneration. To realize this, a bone plate already in clinical use was coated with photo-cross linked Az-CH loaded with a drug, BMP2. In this work, the drug could be released from the coating for approximately 63 days in a sustained manner, maintaining a therapeutically effective drug level. Therefore, when this BMP2-delivery plate was fixed on a calvarial critical size defect in vivo, a statistically significantly higher volume of newly formed bone was observed than those with the plates without the drug. The BMP2-delivery plate also exhibited 
good in vivo bio-compatibility, similar to the Inion plate already in clinical use. Therefore, we conclude that a bone plate enabled with the sustained, local delivery of BMP2 can be a promising system for both bone fixation and its expedited repair.

\section{References}

1. CG Ambrose, TO Clanton (2004) Bioabsorbable implants: review of clinical experience in orthopedic surgery. Ann Biomed Eng 32(1): 171177.

2. PB Maurus, CC Kaeding (2004) Bioabsorbable implant material review. Oper Techn Sport Med 12: 158-160.

3. JF Keating, AH RW Simpson, CM Robinson (2005) the management of fractures with bone loss. J Bone Joint Surg 87(2): 142-150.

4. RB Gustilo, JT Anderson (1976) Prevention of infection in the treatment of one thousand and twenty-five open fractures of long bones: retrospective and prospective analyses. J Bone Joint Surg Am 58(4): 453-458.

5. RH Quinn, DJ Macias (2006) the management of open fractures. Wilderness Environ Med 17(1): 41-48.

6. JP Rodriguez, S Garat, H Gajardo, AM Pino, G Seitz (1999) abnormal osteogenesis in osteoporotic patients is reflected by altered mesenchymal stem cells dynamics. J Cell Biochem 75(3): 414-423.

7. JG Liu, XX Xu (1994) Stress shielding and fracture healing. Zhonghua Yi Xue Za Zhi 74(8): 483-485,519.

8. PV Giannoudis, E Schneider (2006) Principles of fixation of osteoporotic fractures. J Bone Joint Surg 88B: 1272-1278.

9. M Stover (2001) distal femoral fractures: current treatment, results and problems. Injury 3: 3-13.

10. K Stromsoe (2004) Fracture fixation problems in osteoporosis. Injury 35(2): 107-113.

11. Boden SD, Kang J, Sandhu H, Heller JG (2002) Use of recombinant human bone morphogenetic protein-2 to achieve posterolateral lumbar spine fusion in humans - A prospective, randomized clinical pilot trial - 2002 Volvo Award in clinical studies. Spine 27(23): 2662-2673.

12. McKay WF, Peckham SM, Badura JM (2007) A comprehensive clinica review of recombinant human bone morphogenetic protein-2 (INFUSE Bone Graft). International orthopaedics 31(6): 729-734.

13. Carragee EJ, Hurwitz EL, Weiner BK (2011) A critical review of recombinant human bone morphogenetic protein-2 trials in spinal surgery: emerging safety concerns and lessons learned. Spine J 11(6): 471-491.

14. Smucker JD, Rhee JM, Singh K, Yoon ST, Heller JG (2006) Increased swelling complications associated with off-label usage of rhBMP-2 in the anterior cervical. spine 31(24): 2813-2819.

15. Garrett MP, Kakarla UK, Porter RW, Sonntag VK (2010) Formation of painful seroma and edema after the use of recombinant human bone morphogenetic protein-2 in posterolateral lumbar spine fusions. Neurosurgery 66(6): 1044-1049.

16. Deutsch H (2010) High-dose bone morphogenetic protein-induced ectopic abdomen bone growth. Spine J 10(2): e1-4.

17. Shields LB, Raque GH, Glassman SD, Campbell M, Vitaz T, et al. (2006) adverse effects associated with high-dose recombinant human bone morphogenetic protein-2 use in anterior cervical spine fusion. Spine Phila Pa 31(5): 542-547.

18. Zhang S, Kucharski C, Doschak MR, Sebald W, Uludag H (2010) Polyethylenimine-PEG coated albumin nanoparticles for BMP-2 delivery. Biomaterials 31(5): 952-963.

19. Kirby GTS, White LJ, Rahman CV, Cox HC, Qutachi O, et al. (2011) PLGA-
Based Microparticles for the Sustained Release of BMP-2. PolymersBasel 3(1): 571-586.

20. Geiger M, Li RH, Friess W (2003) Collagen sponges for bone regeneration with rhBMP-2. Advanced drug delivery reviews 55(12): 1613-1629.

21. Li L, Zhou G, Wang Y, Yang G, Ding S, et al. (2015) Controlled dual delivery of BMP-2 and dexamethasone by nanoparticle-embedded electrospun nanofibers for the efficient repair of critical- sized rat calvarial defect. Biomaterials 37: 218-229.

22. Shi P, Abbah SA, Saran K, Zhang Y, Li J, et al. (2013) Silk fibroin-based complex particles with bioactive encrustation for bone morphogenetic protein 2 delivery. Biomacromolecules 14(12): 4465-4474.

23. Kundu B, Rajkhowa R, Kundu SC, Wang X (2013) Silk fibroin biomaterials for tissue regenerations. Advanced drug delivery reviews 65(4): 457 470 .

24. N Bhattarai, J Gunn, MQ Zhang (2010) Chitosan-based hydrogels for controlled, localized drug delivery. Adv Drug Deliv Rev 629(1): 83-99.

25. K Obara, M Ishihara, Y Ozeki, T Ishizuka, T Hayashi, et al. (2005) Controlled release of paclitaxel from photocrosslinked chitosan hydrogels and its subsequent effect on subcutaneous tumor growth in mice. J Control Release 110(1): 79-89.

26. AP Zhu, M Zhang, J Wu, J Shen (2002) Covalent immobilization of chitosan/heparin com- plex with a photosensitive hetero-bifunctional crosslinking reagent on PLA surface. Biomaterials 23(23): 4657-4665.

27. S Aiba, N Minoura, K Taguchi, Y Fujiwara (1987) covalent immobilization of chitosan derivatives onto polymeric film surfaces with the use of a photosensitive hetero-bi- functional cross-linking reagent. Biomaterials 8(6): 481-488.

28. Y Yeo, JA Burdick, CB Highley, R Marini, R Langer, et al. (2006) Peritoneal application of chitosan and UV-cross-linkable chitosan. J Biomed Mater Res A 78(4): 668-675

29. MP Lutolf, FE Weber, HG Schmoekel, JC Schense, T Kohler, et al. (2003) Repair of bone defects using synthetic mimetics of collagenous extracellular matrices. Nat Biotechnol 21(5): 513-518.

30. Woojune Hu, Min Park, Jae Yeon Lee, Myung Hun Kim, Seung Ho Lee, et al. (2016) Bioabsorbable bone plates enabled with local, sustained delivery of alendronate for bone regeneration. Journal of controlled release 222: 97-106.

31. GJ Buijs, EB Van Der Houwen, B Stegenga, RR Bos, GJ Verkerke (2007) Mechanical strength and stiffness of biodegradable and titanium osteofixation systems. J Oral Maxillofac Surg 65(11): 2148-2158.

32. SK Al Deeb, II Hamdan, SM Al Najjar (2004) Spectroscopic and HPLC methods for the determination of BMP2 in tablets and urine. Talanta 64(3): 695-702.

33. Y Huang, S Onyeri, M Siewe, A Moshfeghian, SV Madihally (2005) In vitro characterization of chitosan-gelatin scaffolds for tissue engineering. Biomaterials 26(36): 7616-7627.

34. Z Huang, H Lee, E Lee, SK Kang, JM Nam, et al. (2011) Responsive nematic gels from the self-assembly of aqueous nanofibres. Nat Commun 2: 459.

35. PP Spicer, JD Kretlow, S Young, JA Jansen, FK Kasper, et al. (2012) Evaluation of bone regeneration using the rat critical size calvarial defect. Nat Protoc 7(10): 1918-1929.

36. CG Park, C Shasteen, Z Amoozgar, J Park, SN Kim, et al. (2015) Photocrosslinkable chitosan hydrogel as a bioadhesive for esophageal stents. Macromol Res 23(9): 1-6.

37. J Hankiewi, E Swiercze (1974) Serum in human body-fluids. Clin Chim Acta 57: 205-209.

38. Y Huang, S Onyeri, M Siewe, A Moshfeghian, SV Madihally (2005) In vitro characterization of chitosangelatin scaffolds for tissue engineering. Biomaterials 26(36): 7616-7627. 
39. T Nieminen, I Rantala, I Hiidenheimo, J Keranen, H Kainulainen, et al. (2008) Degradative and mechanical properties of a novel resorbable plating system during a 3-year follow- up in vivo and in vitro. J Mater Sci Mater Med 19(3): 1155-1163.

40. Y Ma, Y Zheng, K Liu, G Tian, Y Tian, et al. (2010) Nanoparticles of poly(lactide-co-glycolide)-da-tocopheryl polyethylene glycol 1000 succinate random copolymer for cancer treatment. Nanoscale Res Lett 5(7): 1161-1169.

41. LL Fernandes, CX Resende, DS Tavares, GA Soares, LO Castro, et al. (2011) Cytocompatibility of chitosan and collagen-chitosan scaffolds for tissue engineering. Polímeros 21: 1-6.

42. CY Wang, XF Xiao, D Mao, HZ Tang, RF Liu (2011) the study of using TiO2 nanotube arrays as a drug delivery for BMP2. Adv Mater Res 335-336. 1469-1472.

43. AU Daniels, MK Chang, KP Andriano (1990) Mechanical properties of biodegradable polymers and composites proposed for internal fixation of bone. J Appl Biomater 1(1): 57-78.

44. C Chatelet, O Damour, A Domard (2001) Influence of the degree of acetylation on some biological properties of chitosan films. Biomaterials 22(3): 261-268.

45. Y Xiong, H Yang, J Feng, Z Shi, L Wu (2009) Effects of BMP2 on the proliferation and osteogenic differentiation of $\mathrm{C} 2 \mathrm{C} 12$ cells. J Int Med Res 37: 407-416.

46. SD Patil, F Papadmitrakopoulos, DJ Burgess (2007) Concurrent delivery of dexamethasone and VEGF for localized inflammation control and angiogenesis. J Control Release 117(1): 68-79.

47. T Hickey, D Kreutzer, DJ Burgess, F Moussy (2002) In vivo evaluation of a dexamethasone/PLGA microsphere system designed to suppress the inflammatory tissue response to implantable medical devices. J Biomed Mater Res 61(2): 180-187.

48. CZ Wang, SM Chen, CH Chen, CK Wang, GJ Wang, et al. (2010) the effect of the local delivery of BMP2 on human adipose-derived stem cell-based bone regeneration. Biomaterials 31(33): 8674-8683.

49. SJ Meraw, CM Reeve, PC Wollan (1999) Use of BMP2 in peri-implant defect regeneration. J Periodontol 70: 151-158.

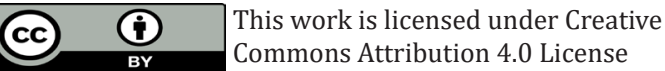

Submission Link: http://biomedres.us/submit-manuscript.php
50. JA Cottrell, FM Vales, D Schachter, S Wadsworth, R Gundlapalli, et al. (2010) osteogenic activity of locally applied small molecule drugs in a rat femur defect model. J Biomed Biotechnol 2010: 597641.

51. H Schell, J Lienau, D Epari, P Seebeck, C Exner, S Muchow, et al. (2006) Osteoclastic activity begins early and increases over the course of bone healing. Bone 38(4): 547-554.

52.XZ Shu, KJ Zhu, W Song (2001) Novel pH-sensitive citrate cross-linked chitosan film for drug controlled release. Int J Pharm 212(1): 19-28.

53. R Pauliukaite, ME Ghica, O Fatibello Filho, CM Brett (2009) Comparative study of different crosslinking agents for the immobilization of functionalized carbon nanotubes within a chitosan film supported on a graphite-epoxy composite electrode. Anal Chem 81(13): 5364-5372.

54.VL Gonçalves, M Laranjeira, VT Fávere, RC Pedrosa (2005) Effect of crosslinking agents on chitosan microspheres in controlled release of diclofenac sodium. Polimeros 15: 6-12.

55. M Ishihara, M Fujita, K Obara, H Hattori, S Nakamura, et al. (2006) B Takase, M Kikuchi, Controlled releases of FGF-2 and paclitaxel from chitosan hydrogels and their subsequent effects on wound repair, angiogenesis, and tumor growth. Curr Drug Deliv 3(4): 351-358.

56. HG Bone, S Adami, R Rizzoli, M Favus, PD Ross, et al. (2000) Weekly administration of BMP2: rationale and plan for clinical assessment. Clin Ther 22(1): 15-28.

57. T Van den Wyngaerta, MT Huizing, JB Vermorken (2007) Osteonecrosis of the jaw related to the use of bisphosphonates. Curr Opin Oncol 19: 315-322.

58.S Srisubut, A Teerakapong, T Vattraphodes, S Taweechaisupapong, K Kaen (2007) Effect of local delivery of BMP2 on bone formation in bioactive glass grafting in rats. Oral Surg Oral Med Oral Pathol 104: E11-E16.

59. CW Kim, YP Yun, HJ Lee, YS Hwang, IK Kwon, et al. (2010) In situ fabrication of BMP2- loaded calcium phosphate microspheres: controlled release for inhibition of osteoclastogenesis. J Control Release 147: 45-53.

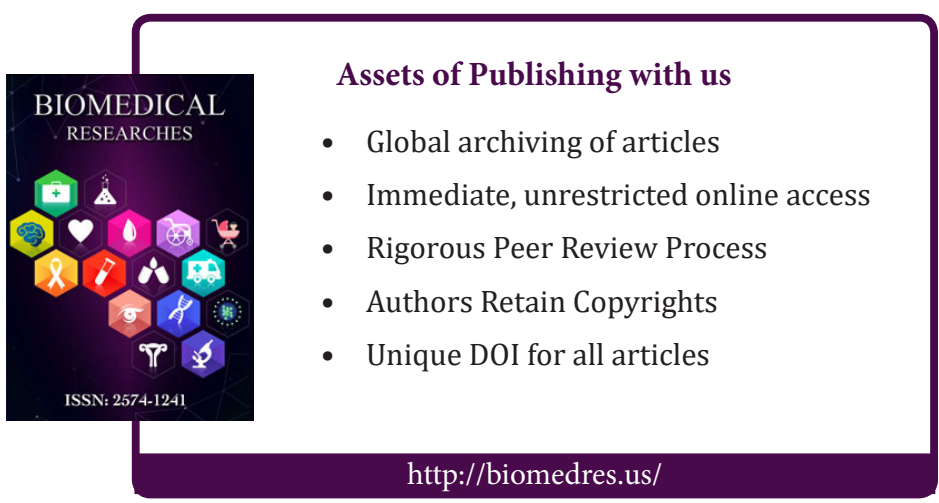

